\title{
Correlação das variáveis para mapeamento de vulnerabilidade aos de- sastres hidrológicos no Arroio Forromeco-RS
}

\author{
Correlation of variables for vulnerability mapping to hydrological disasters in \\ Arroyo Forromeco-RS
}

\author{
Janete Teresinha Reis ${ }^{1}$, Masato Kobiama ${ }^{2}$ Fernando Campo Zambrano ${ }^{3}$ e Gean Paulo Michel ${ }^{4}$ \\ ${ }^{1}$ Pós-doutoranda em Ciências Ambientais, UFRGS, RS - Brasil \\ janete.reis@uffs.edu.br \\ ${ }^{2}$ Prof. Titular do Instituto de Pesquisas Hidráulicas, UFRGS, RS - Brasil \\ masato.kobiyama@ufrgs.br \\ 3,4 Doutorandos em Recursos Hídricos e Saneamento Ambiental - Brasil \\ ing.ambientalfernando@gmai.com; geanpmichel@gmail.com
}

\begin{abstract}
Resumo
A Análise de Correlação é um método estatístico usado para determinar o grau de relacionamento entre duas ou mais variáveis. Assim, fornece um número que identifica como duas variáveis diferem conjuntamente. Com esse propósito, o presente artigo tem como objetivo estabelecer a correlação entre as variáveis que determinaram o indice de vulnerabilidade na bacia hidrográfica do Arroio Forromeco, RS, no intuito de reduzir elou determinar a importância das variáveis para mapeamentos de vulnerabilidade. Para contemplar o objetivo, utilizaram-se dados do Censo do IBGE que permitiram estabelecer o índice de vulnerabilidade por meio das variáveis socioeconômicas. A correlação matricial resultou em quatro cenários distintos, apontando novos cenários para a espacialização de vulnerabilidade a desastres naturais. Estes cenários confirmaram áreas de maior vulnerabilidade na parte nortenordeste da bacia hidrográfica, no município de Farroupilha e os da parte central-norte da bacia hidrográfica, no município de Carlos Barbosa, resultaram em baixa e muito baixa vulnerabilidade. No entanto, a indicação das variáveis apropriadas para o mapeamento de vulnerabilidade permite maior eficácia na intervenção e tomada de decisão frente a prevenção e ocorrência de desastres hidrológicos.
\end{abstract}

Palavras-chave: Correlação; Variáveis; Vulnerabilidade; Desastres Hidrológicos.

\begin{abstract}
The correlation analysis is a statistical method used to determine the degree of relationship between two or more variables. It thus provides a number that identifies how two variables vary together. To that end, this article aims to establish the correlation between the variables that determine the vulnerability index in the basin of Forromeco Arroyo, RS, in order to reduce and / or to determine the importance of the variables for vulnerability mapping. To address the purpose, we used the IBGE Census data allowed to establish the vulnerability index by means of socioeconomic variables. The correlation matrix resulted in four different scenarios, pointing out new scenarios for spatial vulnerability to natural disasters. These scenarios have confirmed most vulnerable areas in the northnortheastern part of the basin, in the municipality of Farrukhabad and the central-northern part of the basin, in the city of Carlos Barbosa, resulted in low and very low vulnerability. However, an indication of the appropriate variables for vulnerability mapping allows more effective intervention and decision making across the prevention and occurrence of hydrological disasters.
\end{abstract}

Keywords: Correlation Variables; Vulnerability; Hydrological Disasters. 


\section{Introdução}

Como ferramenta estatística utilizada para análises científicas, a correlação é de extrema importância por fornecer um número que indica como duas variáveis diferem conjuntamente. Além de medir a intensidade e a direção da relação linear (positiva ou negativa) ou não linear entre duas variáveis. Segundo Lira (2004) a correlação é um indicador que atende à necessidade de se estabelecer a existência ou não de uma relação entre variáveis sem que, para isso, seja necessário o ajuste de uma função matemática. E complementa que não possui distinção entre a variável explicativa e a variável resposta. Em outras palavras, que o grau de variação conjunta entre $X \mathrm{e}$ $\mathrm{Y}$ é igual àquela de variação entre $\mathrm{Y}$ e $\mathrm{X}$.

Ao avaliar o grau de relacionamento entre duas ou mais variáveis, segundo Guimarães (2015) é possível descobrir com precisão, o quanto uma variável interfere no resultado de outra. Portanto, as técnicas associadas à análise de correlação representam uma ferramenta fundamental de aplicação nas Ciências Sociais e do comportamento, da Engenharia e das Ciências Naturais.

Embora, a correlação seja uma técnica antiga, sua representatividade continua destaque em trabalhos atuais. Para o mapeamento de vulnerabilidade considerado uma importante medida não estrutural que complementa o mapeamento de risco, possibilita um conjunto de ações a serem tomadas visando orientar e minimizar os riscos a desastres naturais. Neste sentido, ressaltado Kobiyama et al (2014), deve-se avaliar a eficácia do uso das variáveis nos referidos mapeamentos.

A correlação orientada sob a visão de estabelecer a existência ou não da relação entre as variáveis de vulnerabilidade permite discriminar, orientar e indicar as mais importantes para o mapeamento, além de apontar cenários para tomada de decisão. Em nível municipal possibilita aos técnicos realizarem mapeamentos de vulnerabilidade a desastres naturais de forma rápida e eficiente, desde que os gestores analisam os resultados de forma critica e em coerência com a realidade da área em análise.

Um indicador reflete um fenômeno quantitativamente e pode ser considerado elementar na determinação da capacidade de uma comunidade absorver, enfrentar ou recuperar-se de um desastre. De acordo com Pine (2008) nenhum conjunto de indicadores consegue ser inclusivo totalmente. Embora as discussões sobre perigo, vulnerabilidade e as variáveis a eles associados tenham evoluído, existem desafios a serem desvendados quanto ao mapeamento de vulnerabilidade, principalmente no que se refere às variáveis utilizadas para o mapeamento.

Schenkel et al (2015) realizaram mapeamento das áreas de risco de escorregamentos transnacionais na bacia hidrográfica do Arroio Forromeco, onde as variáveis associadas ao levantamento da vulnerabilidade tiveram como base a metodologia de Goerl et al (2012) e Reis et al (2014). Porém, o presente trabalho vai além ao determinar a importância das variáveis (utilizadas pelos autores) e apresentar diferentes cenários de vulnerabilidade a desastres naturais, a partir da correção positiva e/ou negativas caracterizadas também importantes no mapeamento de risco a desastres naturais.

Segundo Kobiyama et al. (2014), para o gerenciamento desses desastres a fim de reduzi-los é necessário medidas não estruturais que envolvem essencialmente ações voltadas à redução da vulnerabilidade da população, que sejam implementadas juntamente às estruturais. Assim, a realização do mapeamento da vulnerabilidade, uma importante medida não estrutural, pode orientar um conjunto de ações a serem tomadas a fim de minimizar o risco a desastres hidrológicos.

Assim, a definição das variáveis para o mapeamento da vulnerabilidade é de extrema importância, além do aprimoramento metodológico poderá auxiliar as prefeituras municipais na prevenção, planejamento e inclusive na relocação de famílias reduzindo prejuízos materiais e principalmente evitar vítimas fatais. Com este intuito, o presente artigo tem como objetivo estabelecer a correlação entre as variáveis que determinaram o índice de vulnerabilidade na bacia hidrográfica do Arroio Forromeco, RS, e determinar sua importância.

\section{Metodologia}

\section{Área de estudo}

A área de estudo (bacia hidrográfica do Arroio Forromeco), considerada cabeceira da bacia hidrográfica do Rio Caí, localiza-se na porção leste do Rio Grande do Sul, abrangendo os municípios de Alto Feliz, São Vendelino, Barão, Bom Princípio, Carlos Barbosa e Farroupilha. Estudos nessa área são importantes, devido à frequente ocorrência de inundações bruscas e escorregamentos, predominantemente nos trechos alto e médio Caí (Comitê Caí, 2008) e também à de inundações graduais que ocorrem com freqüência no trecho baixo Caí (PEDROLLO et al., 2011). Para isso, um dos elementos indispensáveis consiste em mensurar o índice de vulnerabilidade local.

A área de estudo está situada entre as coordenadas $-51,57 \mathrm{E}-51,16 \mathrm{E}$, e entre $-29,42 \mathrm{~N}$ e $-29,21 \mathrm{~N}$, e possui área total de $290,88 \mathrm{~km}^{2}$. Sua altitude varia de $60 \mathrm{~m}$ até $790 \mathrm{~m}$. (Figura 1). 


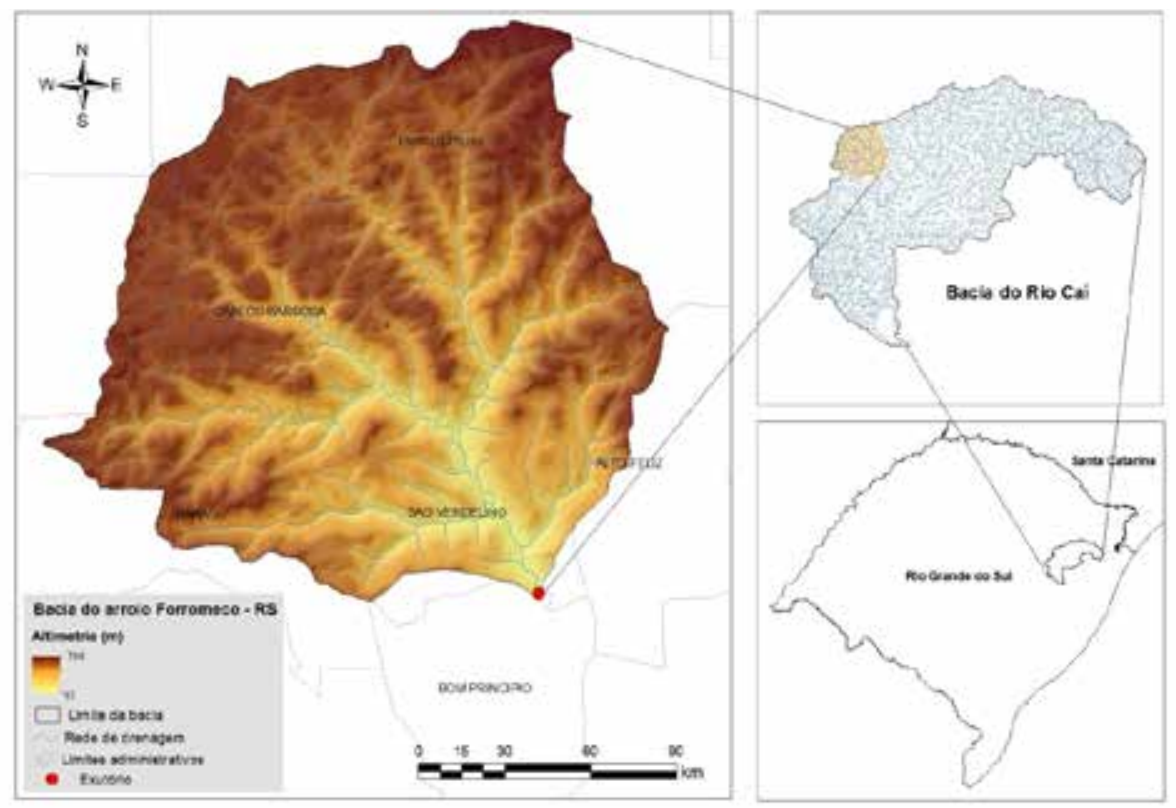

Figura 1 - Localização da bacia hidrográfica do Arroio Forromeco, RS

\section{Variáveis de Vulnerabilidade}

As variáveis de vulnerabilidade foram estimadas a partir de dados socioeconômicos coletados no Censo do Instituto Brasileiro de Geografia e Estatística (IBGE, 2010). As oito variáveis censitárias selecionadas, para determinar o índice de vulnerabilidade podem ser visualizadas na Tabela 1.

As variáveis selecionadas foram as mesmas utilizadas por Goerl et al. (2012) e Reis et al. (2014). Porém, o diferencial dos parâmetros consiste na idade definida para determinação da população dependente. Como unidade de análise, o IBGE identifica e analisa os dados a partir das unidades censitárias, caracterizada como a menor unidade territorial com limites físicos identificáveis a campo. A investigação realizada pelo IBGE (2010), referente às características dos domicílios e das pessoas neles residentes, teve como data de referência o dia 31 de julho de 2010, de acordo com os seguintes critérios:

Tabela 1 - Variáveis censitárias utilizadas para mensurar a vulnerabilidade e a correlação

\begin{tabular}{c|c}
\hline Variáveis censitárias & Variáveis de vulnerabilidade \\
\hline Número de moradores & 1 - Número de moradores no setor \\
\hline Média de moradores por domicílio & 2 - Média de moradores por domicílio \\
\hline Densidade demográfica $\left(\mathrm{hab} / \mathrm{km}^{2}\right)$ & 3- Densidade Demográfica \\
\hline Percentual da população acima de 65 anos & $\begin{array}{c}\text { 4- Soma da porcentagem da população acima de } 65 \\
\text { e abaixo de 10 anos }\end{array}$ \\
\hline Percentual da população abaixo de 10 anos & $\begin{array}{c}\text { 5 - Percentual de pessoas analfabetas acima de 10 } \\
\text { anos }\end{array}$ \\
\hline Percentual de pessoas analfabetas acima de 10 anos & $\begin{array}{c}\text { 6 - Soma da porcentagem dos responsáveis sem } \\
\text { rendimento e com rendimento de até 1 Salário } \\
\text { Percentual de responsáveis sem rendimento }\end{array}$ \\
\hline Percentual de responsáveis com rendimento até 1 & \\
\hline Salário Mínimo &
\end{tabular}

Adaptado de Goerl et al. (2012) e Reis et al. (2014) 
- Pessoa alfabetizada: aquela pessoa capaz de ler e escrever um bilhete simples no idioma que conhece. A pessoa que aprendeu a ler e escrever, mas que esqueceu pelo fato de ter passado por um processo de alfabetização que não se consolidou, e a que apenas assina o próprio nome, foi considerada analfabeta.

- Pessoa responsável: para a pessoa (homem ou a mulher) de 10 anos ou mais de idade, reconhecida pelos moradores como responsável pela unidade domiciliar.

- Rendimento: a soma do rendimento nominal mensal de trabalho com o proveniente de outras fontes.

- Dependência: nesse quesito adotou-se como limite 10 e 60 anos. Os 10 anos porque o Estatuto da Criança e do Adolescente considera as pessoas abaixo dessa idade como crianças, sendo totalmente dependentes, perante a lei e sociedade, de seus pais ou responsáveis. Considerou-se superior a 65 anos porque segundo a Organização Mundial de Saúde, a partir dessa idade as pessoas são consideradas idosas (GOERL, et al. 2012). Esse estatuto tem como propósito tutelar de forma específica os direitos do idoso, estabelecendo direitos e medidas de proteção dessa categoria.

A partir desses quesitos, foram determinadas 6 variáveis que constituíram o Índice de Vulnerabilidade (IV):

$$
I V=\frac{D d+M s+M m+P D+P A+R}{I D H M}
$$

Onde IV é o índice de vulnerabilidade; Dd é a densidade demográfica; Ms é o número de moradores; Mm é a média de moradores por domicílio; PD é o percentual de dependência de idosos e jovens; PA é o percentual de analfabetos acima de 10 anos; R é a renda do responsável sem rendimento e até 1 salário mínimo e IDHM é o índice de desenvolvimento humano. Aqui nota-se que o índice e todas as variáveis exceto IDHM são calculados por setor censitário, sendo que o valor de IDHM é municipal. O IDHM é obtido pela média aritmética de três sub-índices: IDHM longevidade, IDHM educação e IDHM renda (PNUD, 2014).

$\mathrm{O}$ valor de IV foi espacializado concomitantemente com a correlação matricial contemplando as variáveis de forma intercalada apresentando diferentes cenários para tomada de decisão.

Com base ao afirmado e nos conceitos expostos neste trabalho, considera-se que a vulnerabilidade é inversamente proporcional a capacidade de suporte/resposta ou de preparo do município. O valor de IDHM de cada município Alto Feliz São Vendelino, Barão, Bom Princípio, Carlos Barbosa e Farroupilha foi utilizado como indicador dessa capacidade.

Para normalizar as unidades, todas as variáveis foram escalonadas de 0 a 1 . Posteriormente, aplicou-se a Equação (1) e novamente realizou-se a normalização para definir a vulnerabilidade final. $O$ presente trabalho agrupou o IV em cinco classes: 0 a 0,499 (Muito baixo desenvolvimento), 0,5 a 0,599 (Baixo desenvolvimento); 0,6 a 0,699 (Médio desenvolvimento); 0,7 a 0,799 (Alto desenvolvimento); e 0,8 a 1,00 (Muito Alto desenvolvimento).

Com base em Marcelino et al. (2006), a normalização, que varia de 0 a 1 , foi calculada por:

$$
V_{n}=\frac{V_{\mathrm{o}}-V_{\min }}{V_{\text {max }}-V_{\min }}
$$

Onde Vn é o valor normalizado; Vo é o valor observado; Vmín é o valor mínimo; e Vmáx é o valor máximo.

Os valores de IV foram definidos através do método estatístico Natural Breaks nas classes acima mencionadas. Souza et al. (2006) mencionaram que esse método consiste em "minimizar a variância dentro de cada classe pelo método estatístico, fornecendo categorias que apresentam valores mais homogêneos possíveis dentro das classes".

A base de dados da área de estudo foi extraída de Schenkel (2015).

\section{Correlação matricial das variáveis de vulnera- bilidade}

As variáveis coletadas, junto ao IBGE (2010), para análise da correlação matricial, foram tabuladas e codificadas em planilha de Excel, posteriormente inseridas, processadas e analisadas com recursos do programa Basic Statistic.

A correlação matricial foi realizada contemplando as variáveis de forma intercalada, por meio do ranqueamento possibilitando projetar diferentes cenários para tomada de decisão. Desse modo, a correlação linear das variáveis apresentada na Tabela 1, a citar, Ms, Mm, Dd, PD, PA, e R, contempla o conjunto das informações coletadas junto ao IBGE, foram espacializadas por meio de software livre Quantum Gis 2.8.1 e processadas no software Statistic.

Após a correlação matricial, ao constatar significativa correlação positiva entre algumas variáveis foram gerados diferentes cenários visando identificar as áreas mais vulneráveis e compará-las com o mapa de vulnerabilidade da integração de todas as variáveis adotadas no trabalho anteriormente.

\section{Resultados}

\section{Espacialização integrada}

A espacialização do IV por meio da integração de todas as variáveis permitiu a análise sem discriminar 


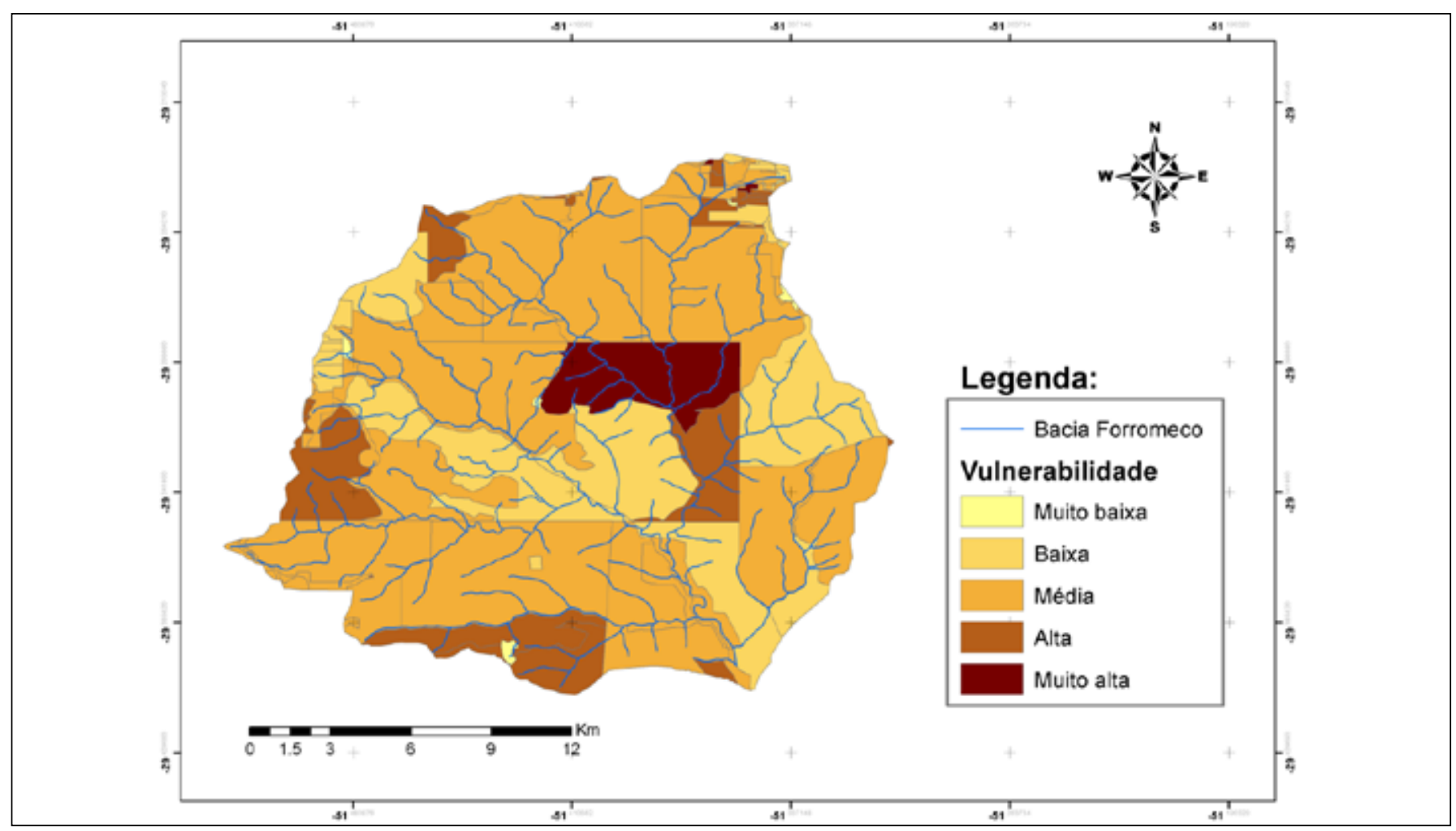

Figura 2 - Espacialização do índice de vulnerabilidade na bacia hidrográfica do Arroio Forromeco, RS

ou estabelecer a importância entre as variáveis socioeconômicas. Assim, antes de estabelecer a correlação matricial, todas as variáveis possuem o mesmo nível de importância identificando as unidades censitárias que apresentam maior e menor valor de IV.

Portanto, o setor com maior valor de IV da bacia hidrográfica do arroio Forromeco está situado no município de Carlos Barbosa, como muito alta vulnerabilidade e pequenas áreas do município de Farroupilha (Figura 2).

\section{Correlação das variáveis}

Um primeiro passo comum das análises de dados que envolvem inúmeras variáveis consiste em gerar uma matriz de correlação de todas as variáveis. Assim, a matriz de correlação foi gerada para todas as variáveis para examinar as relações de significância esperada. Com o objetivo científico de encontrar relações entre variáveis, as cores vermelhas significam que possuem correlação positiva, ou negativa. Assim, a variável Ms apresenta significativa relação positiva com $\mathrm{Dd}$, uma significância de 0,58, ou seja, representa 58\% de significância (Tabela 3). Já com as demais variáveis apresenta correlação negativa. Destaca-se a forte correlação negativa da variável, Ms com o PA perfazendo um total de $-0,44$. As combinações que demonstraram a significativa correlação negativa são Ms - PA, Ms - R, e Dd - R.

A variável, Ms demonstra significativa correlação positiva com a Dd, já com relação a PA apresenta correlação negativa, em torno de $44 \%$. A Figura 3 demonstra esta significativa correlação positiva entre as variáveis, Ms e Dd.

Tabela 3 - Correlação matricial entre todas as variáveis em análise

\begin{tabular}{lcccccc}
\hline Variável & $M s$ & $M m$ & $D d$ & $P A$ & $R$ & $P D$ \\
\hline$M s$ & 1,00 & $-0,10$ & 0,58 & $-0,44$ & $-0,22$ & 0,01 \\
$M m$ & $-0,10$ & 1,0 & 0,05 & 0,18 & $-0,03$ & 0,05 \\
$D d$ & 0,58 & 0,05 & 1,00 & $-0,17$ & $-0,23$ & $-0,06$ \\
$P A$ & $-0,44$ & 0,18 & $-0,17$ & 1,00 & 0,36 & 0,02 \\
$R$ & $-0,22$ & $-0,03$ & $-0,23$ & 0,36 & 1,00 & 0,02 \\
$P D$ & 0,01 & 0,05 & $-0,06$ & 0,02 & 0,02 & 1,00 \\
\hline
\end{tabular}




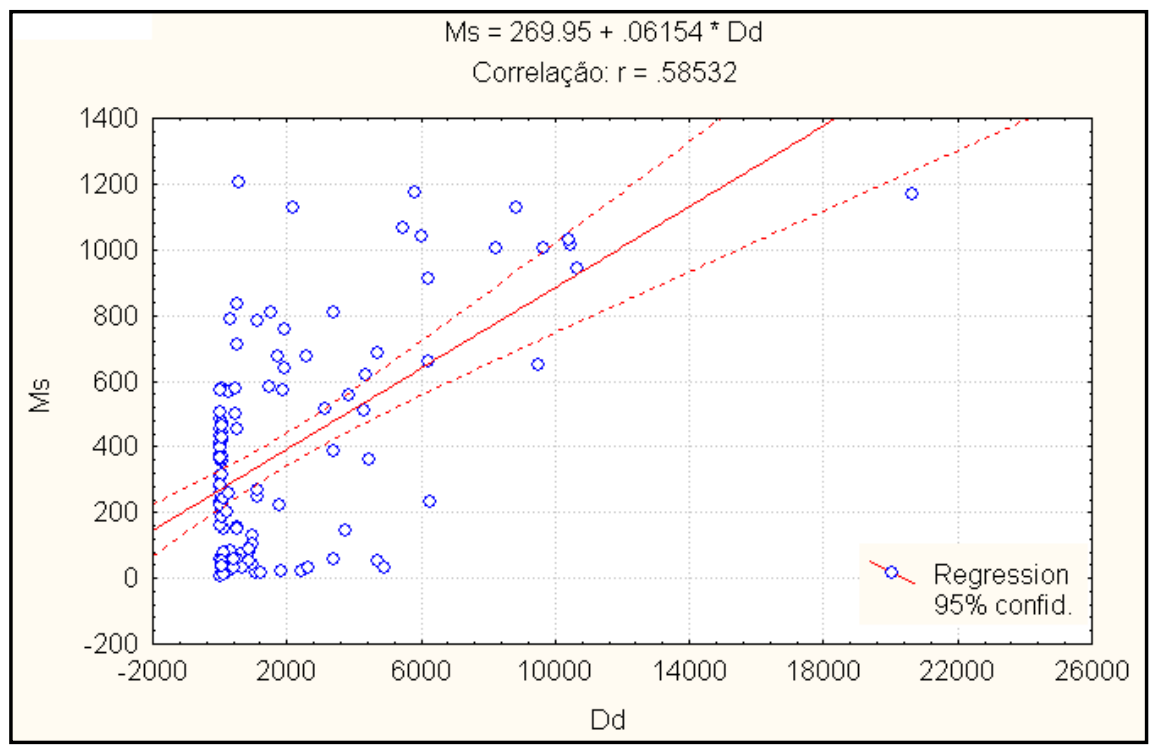

Figura 3 - Correlação matricial positiva das variáveis, Ms e Dd

A referida constatação demonstra que somente uma das variáveis se faz necessária ao determinar o índice de vulnerabilidade, tendo em vista que apresentam redundância e não são determinantes no apontamento dos locais de maior vulnerabilidade. Assim, há possibilidade de inclusão de outras variáveis mais significativas que resultam em maior confiabilidade e veracidade frente à espacialização da vulnerabilidade voltada a desastres naturais na bacia hidrográfica do Arroio Forromeco.

Nesta mesma perspectiva, as variáveis PA e R também apresentaram correlação positiva de forma significativa, porém, menos significativa que a anterior, em torno de $36 \%$ (Tabela 3), o que pode ser evidenciado por meio da Figura 4.

Neste contexto, os estudos de correlação, geralmente, são realizados a partir de dois princípios básicos que visam: às relações e o comportamento entre as variáveis.

As variáveis que apresentam significativa correlação negativa não são determinantes para indicar locais de vulnerabilidade, tendo em vista que sobrepõe a informação frente ao resultado final da espacialização. $\mathrm{Na}$ Figura 5 observa-se uma correlação negativa. O nível de significância é de aproximadamente $44 \%$ entre a variável da PA e Ms para a bacia hidrográfica do Arroio Forromeco, RS.

Ao determinar a correlação matricial entre $\mathrm{R}$ e $\mathrm{Dd}$ constatou-se uma correlação negativa de $23 \%$ (Figura 6). Este resultado, evidência que as referidas variáveis apresentam alguma relação, mas em menor percentual e podem ser consideradas no mapeamento da vulnerabilidade. Outras variáveis que mantiveram correlação negativa foram: R e Ms com 22\%, (Figura 7).

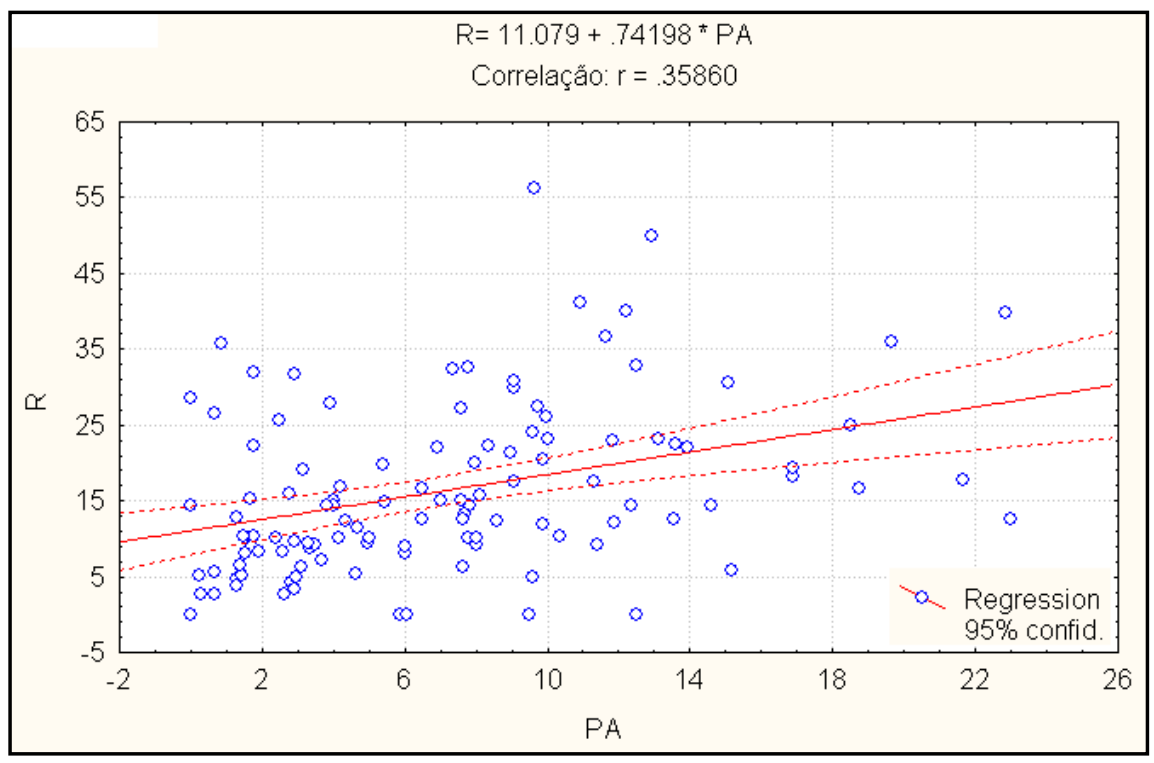

Figura 4 - Correlação matricial positiva das variáveis PA e R 


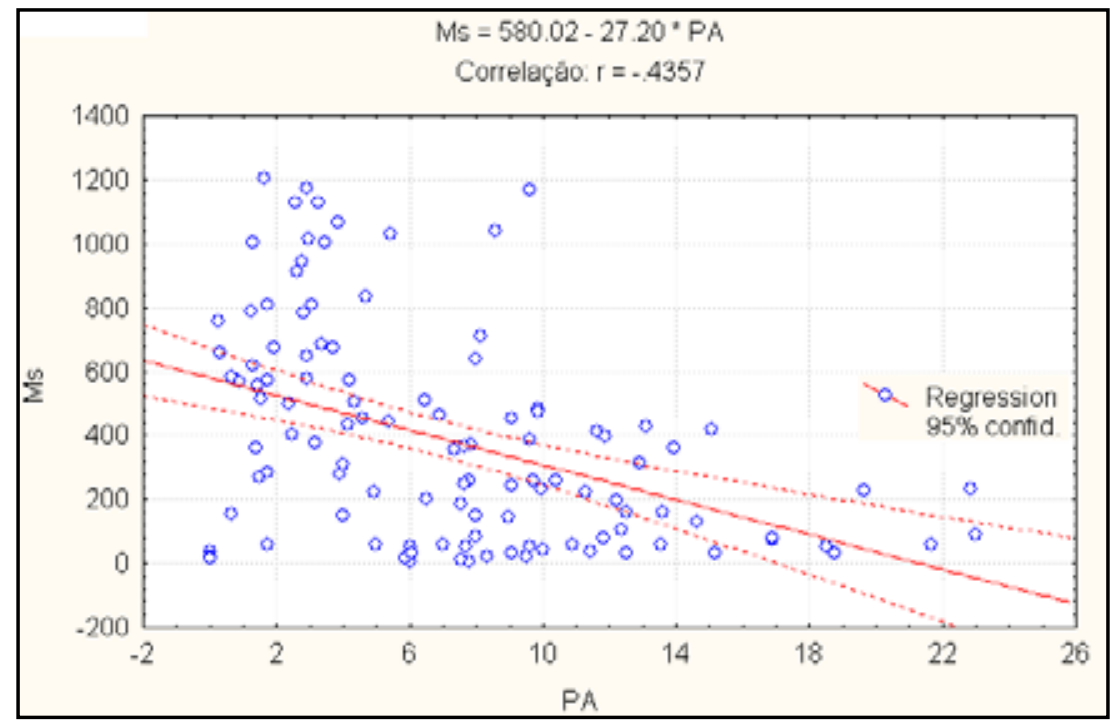

Figura 5 - Correlação matricial negativa das variáveis PA e Ms

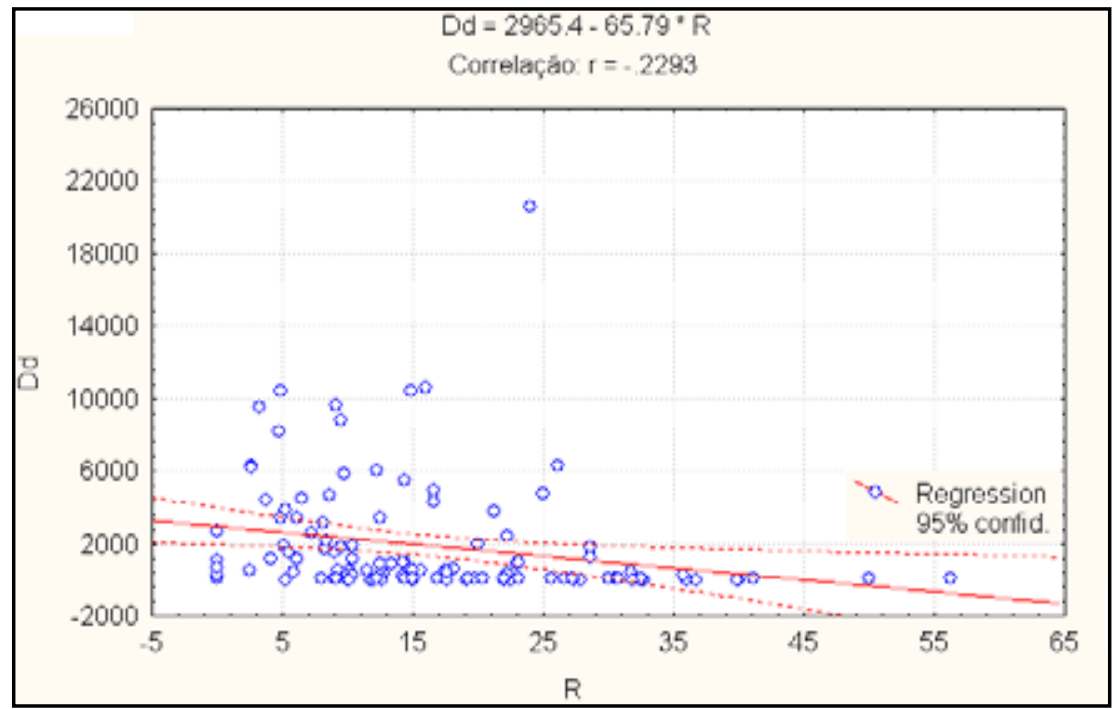

Figura 6 - Correlação matricial negativa das variáveis, R e Dd

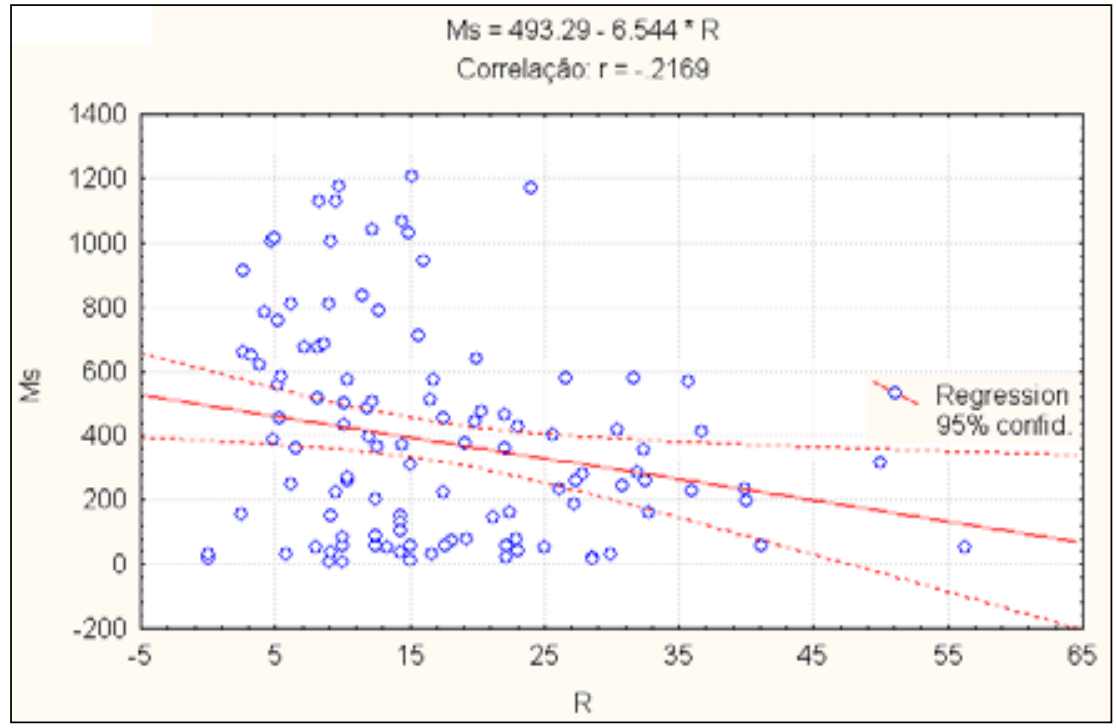

Figura 7- Correlação matricial negativa das variáveis, R e Ms 


\section{Cenários de Vulnerabilidade}

Assim, elaborou-se quatro cenários, onde as variáveis seguiram o seguinte raciocínio: ao adotar a variável Ms, a variável Dd foi excluída (cenário 1), em virtude de apresentarem significativa correlação positiva. Segue o mesmo procedimento para as variáveis PA e R que também apresentaram correlação positiva, porém em menor percentual (Tabela 2). .

Tendo como propósito reduzir as variáveis e/ou determinar sua importância para o mapeamento de vulnerabilidade, ao adotar uma das variáveis de significativa correlação positiva desconsidera a outra. Com este propósito, as análises estatísticas entre as variáveis, resultaram em correlações positivas que originaram diferentes cenários, os quais foram espacializados de acordo com o grau de vulnerabilidade.

Portanto, ao adotar a variável Ms não se faz necessário inserir a variável $\mathrm{Dd}$, pois são redundantes (cenário 1). O mesmo princípio deve ser adotado para as variáveis R e PA (cenário 3), basta incorporar uma das variáveis para efetuar a espacialização da vulnerabilidade a desastres hidrológicos.

Considerando a correlação positiva e redundância de duas variáveis, foram elaborados inicialmente dois cenários diferentes na espacialização do IV conforme resultado da correlação matricial. No Cenário 1 excluiu-se a variável Dd e permaneceu a variável Ms. No Cenário 2, excluiu-se a variável Ms e incorporou Dd, seguem os cenários da espacialização das áreas de vulnerabilidade na bacia hidrográfica do arroio Forromeco, (Figura 8).

No cenário 1 a maior porção da bacia hidrográfica apresenta IV média, enquanto que no cenário 2 certa de $95 \%$ da área apresenta IV muito baixa. Por outro lado, constata-se semelhança no município de Farroupilha, entre dois setores censitários onde o IV é muito alto e alto. Desse modo, ao comparar o Cenário 1 e 2 pela hierarquia, a porção norte-nordeste do município de Farroupilha (destacada em círculo) deveria sofrer intervenção na prevenção de desastres hidrológicos.

No Cenário 3 excluiu-se a variável PA e permaneceu a variável R. No Cenário 4, excluiu-se a variável $\mathrm{R}$ e incorporou PA, seguem os cenários da espacialização das áreas de vulnerabilidade na bacia hidrográfica do arroio Forromeco (Figura 9).

No cenário 3 a maior porção da bacia hidrográfica apresenta IV média, enquanto que no cenário 4 a maior parte apresenta IV muito baixa (Figura 9). Quanto a espacialização do IV constatou-se semelhança entre os Cenários 1 e 4 que apresentaram maior área com IV média, enquanto que os Cenários 2 e 3 apresentaram maior percentual da área como IV muito baixa.

A partir destes cenários é possível verificar, os setores mais vulneráveis e compará-los com o mapeamento de vulnerabilidade estabelecida inicialmente, onde foram adotadas as 8 variáveis, conforme a metodologia proposta.

Portanto, após a correlação matricial, com a exclusão de variáveis observou-se que o setor de maior IV da bacia hidrográfica do Arroio Forromeco não se localiza no município de Carlos Barbosa, na porção central da bacia hidrográfica, tanto na análise do Cenário 1 e 2, quanto nos Cenário 3 e 4 . Por outro lado, as áreas de maior vulnerabilidade que se situavam na porção norte-nordeste do município de Farroupilha podem ser confirmadas em todos os cenários, registrado também, anteriormente a realização da correlação matricial (Figura 2).

Neste contexto, algumas variáveis devem ser mantidas, enquanto que outras devem ser excluídas, entre as excluídas são Ms e PA por também, apresentarem significativa correlação negativa. As demais devem ser mantidas e utilizadas na espacialização da vulnerabilidade a desastres hidrológicos. Desse modo, as variáveis Mm e PD são as mais significativas que devem ser mantidas, além da variável Ms.

A partir dos resultados e discussão sugere-se um novo cenário para a espacialização da vulnerabilidade, que é a integração das variáveis Mm, PD e MS consideradas elementares, pois não apresentaram correlação positivo tão pouca correlação negativa. Portanto, as variáveis, Ms e PA potencializaram a representação do IV na bacia hidrográfica do Arroio Forromeco.

Tabela 2: Cenários gerados a partir da correlação positiva, após aplicação estatística.

\begin{tabular}{c|c|c|c|c}
\hline $\begin{array}{c}\text { Variáveis de } \\
\text { vulnerabilidade }\end{array}$ & Cenário 1 & Cenário 2 & Cenário 3 & Cenário 4 \\
\hline $\mathrm{Ms}$ & $\mathrm{X}$ & & $\mathrm{X}$ & \\
\hline $\mathrm{Mm}$ & $\mathrm{X}$ & $\mathrm{X}$ & $\mathrm{X}$ & $\mathrm{X}$ \\
\hline $\mathrm{Dd}$ & & $\mathrm{X}$ & & $X$ \\
\hline $\mathrm{PD}$ & $\mathrm{X}$ & $\mathrm{X}$ & $\mathrm{X}$ & $\mathrm{X}$ \\
\hline $\mathrm{PA}$ & $\mathrm{X}$ & & & $X$ \\
\hline $\mathrm{R}$ & & $X$ & $X$ & \\
\hline
\end{tabular}




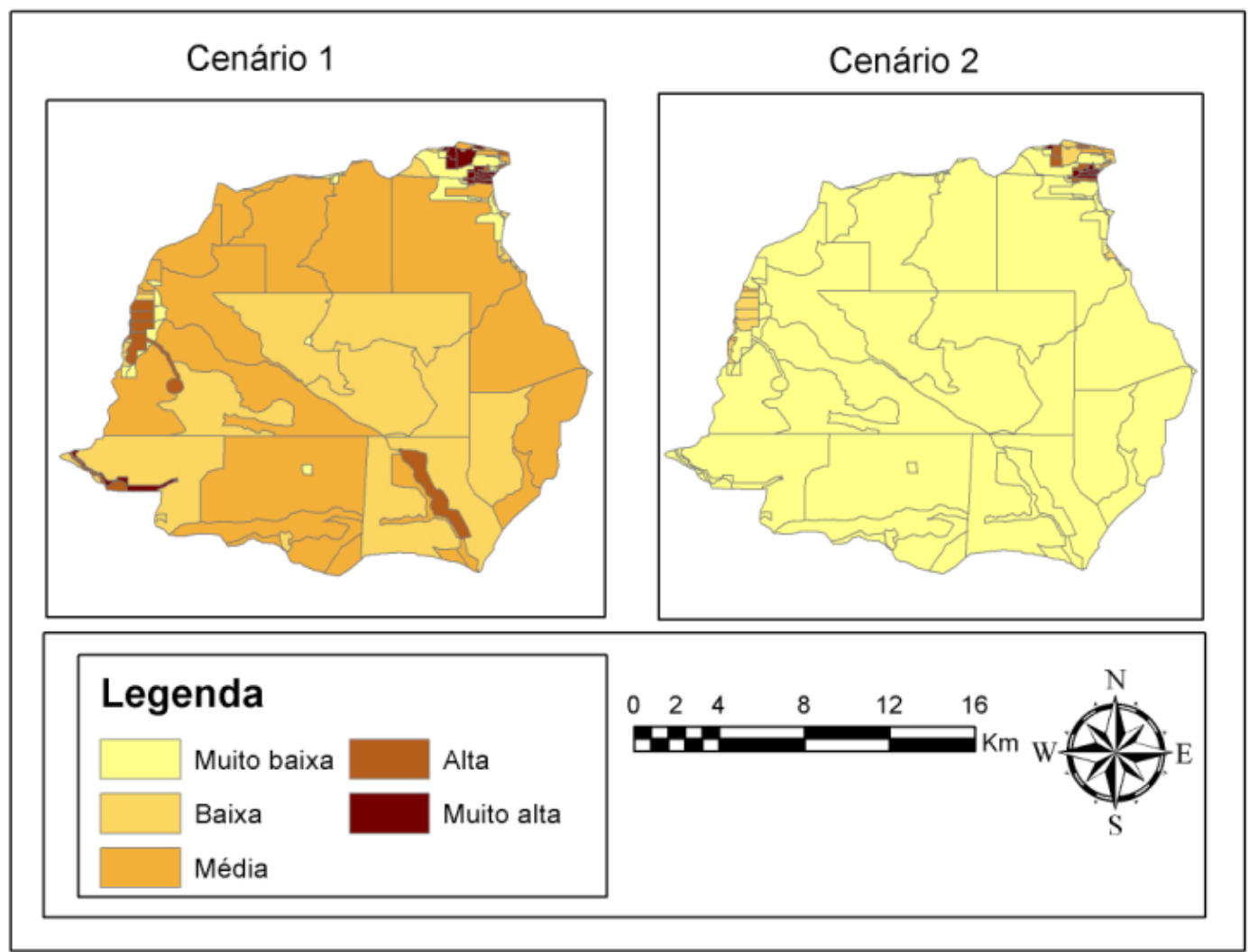

Figura 8- Espacialização do índice de vulnerabilidade na bacia hidrográfica do Arroio Forromeco com os Cenários 1 e 2.

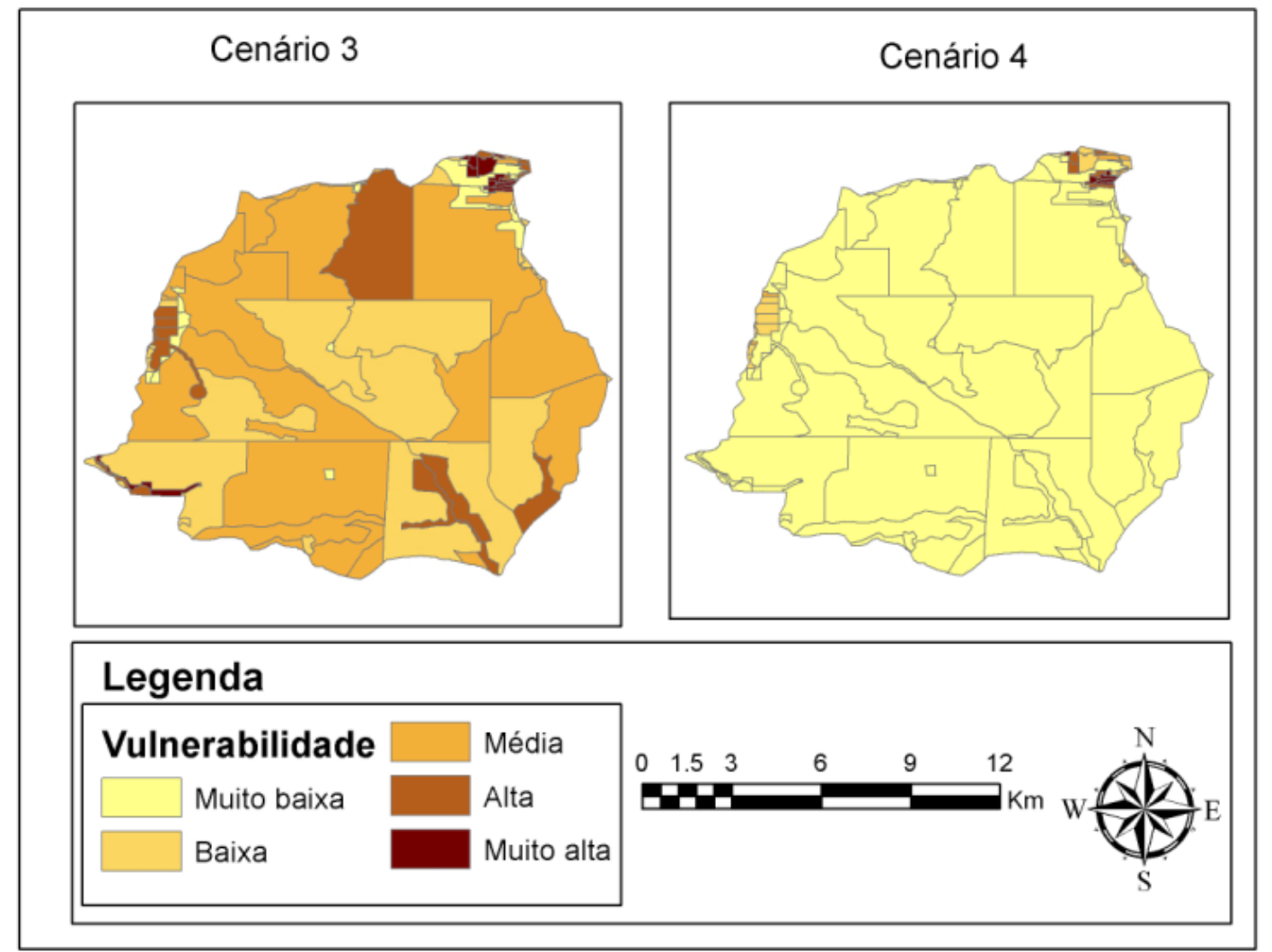

Figura 9 - Espacialização do índice de vulnerabilidade na bacia hidrográfica do Arroio Forromeco com os Cenários 3 e 4 


\section{Conclusões}

O presente artigo teve como objetivo estabelecer a correlação entre as variáveis que determinaram o índice de vulnerabilidade na bacia hidrográfica do Arroio Forromeco, RS. A correlação das variáveis utilizadas para espacialização da vulnerabilidade foram definidas e, portanto, determinaram sua importância, por meio da correlação matricial positiva e negativa.

Ao executar a correlação de todas as variáveis e em seguida examiná-la para relações mais significativas, esperadas e inesperadas, constatou-se duas correlações matriciais positivas que se transformaram em quatro cenários. As variáveis, Ms e Dd nos (Cenários 1 e 2) assim como, PA e R (Cenários 3 e $4)$, ambas remetem somente a uma das variáveis necessárias na espacialização da vulnerabilidade. Já as variáveis, PA e Ms apresentaram significativa correlação negativa em torno de $44 \%$, e, assim inviabilizam sua permanência na espacialização do IV.

O que se percebe é que, a sobreposição de variáveis que mantém significativa correlação positiva mascara a realidade, e assim não comprova a veracidade das áreas de maior vulnerabilidade, frente aos desastres entre os setores espacializados. Portanto, o resultado da correlação matricial, por meio da validação das variáveis trouxe informações importantes para espacialização da vulnerabilidade, com um objetivo específico, a não sobreposição das variáveis com significativa correlação positiva e negativa.

Em suma, a presente pesquisa estabeleceu correlações entre variáveis que comprovam seu nível de significância. Com isto, almeja-se suporte a tomada de decisão por meio da espacialização de locais mais e/ou menos vulneráveis a desastres hidrológicos tendo em vista que, este tipo de desastre vem se intensificando no Rio Grande do Sul e requer pesquisas científicas mais profundas como forma de prevenção.

Diante disso, sugere-se um novo cenário para a espacialização da vulnerabilidade, que é a integração das variáveis Mm, PD e Ms consideradas elementares na espacialização do IV e a incorporação de variáveis para estabelecer a correlação matricial.

\section{Agradecimentos}

Ao Conselho Nacional de Desenvolvimento Científico e Tecnológico/CNPq pelo auxílio na Pesquisa do projeto "Aperfeiçoamento metodológico de mapeamento de risco a desastres naturais na Bacia hidrográfica do Rio Caí/RS".

\section{Referências}

BRASIL. Estatuto da Criança e do Adolescente. (1990). Lei n. 8.069/90. Disponível em: <http://presrepublica. jusbrasil.com.br/legislacao/91764/estatuto-da-criancae-do-adolescente-lei-8069-90\#art-60>. Acesso em: 12 set. 2014.

BRASIL. Estatuto do Idoso. (2003). Lei n. 10.741/03. Disponível em: <http://www.planalto.gov.br/ccivil_03/ leis/2003/110.741.htm>. Acesso em: 20 set. 2014.

GOERL, R. F.; KOBIYAMA, M.; PELLEIN, J.R.G.M. (2012). Proposta metodológica para mapeamento de áreas de risco a inundação: Estudo de caso do município de Rio Negrinho - SC. Boletim de Geografia (UEM) 30(1), pp.81-100.

GUIMARÃES, P. R.B. Análise de Correlação e medidas de associação. Disponível em: http://people.ufpr.br/ jomarc/ correlacao.pdf. Acesso em: 07/set/2015.

ÍNDICE DE DESENVOLVIMENTO HUMANO MUNICIPAL. (2014). Atlas do desenvolvimento humano no Brasil. Disponível em: <http://www.atlasbrasil.org. br/2013/pt/o_atlas/idhm/>. Acesso em: 04 out. 2014.

INSTITUTO BRASILEIRO DE GEOGRAFIA E ESTATÍSTICA - IBGE. (2010). Malhas digitais - Censo 2010 - Unidades censitárias. Disponível em: <http:// downloads.ibge.gov.br/downloads_geociencias.htm>. Acesso em: 12 maios 2014.

KOBIYAMA, M.; MICHEL, G.P.; SILVA, J.S.; REIS, J.T.; MOTA, A.A.; MONTEIRO, L.R. Mapeamento de Áreas de Risco para Prevenção de Desastres Hidrológicos com Ênfase em Modelagem Hidrogeomorfológica. Porto Alegre: UFRGS/IPH/GPDEN, 2014. 466p.

LIRA, S. A. Análise de correlação: abordagem teórica e de construção dos coeficientes com aplicações. Universidade Federal do Paraná. Dissertação de Mestrado - Curso de Pós-graduação em Métodos Numéricos em Engenharia dos Setores de Ciências Exatas e de Tecnologia da Universidade Federal do Paraná: CURITIBA, 2004.

MARCELINO, E.V.; NUNES, L.H.; KOBIYAMA, M. (2006). Mapeamento de risco de desastres naturais do estado de Santa Catarina. Caminhos da Geografia (UFU), Uberlândia, v.7, n.17, 72-84.

PEDROLLO, M.; GERMANP, A.; SOTÉRIO, P.; RODRGIQUES, É.; MADUELL, J.C. Alerta hidrológico da bacia do rio Caí: concepção e implantação do sistema. In: XIX Simpósio Brasileiro de Recursos Hídricos. Maceió: ABRH, Anais, 2011. 14p. 
PINE, J. C. Natural Hazard Analysis: Reducing the impact of disasters. Florida: Taylor \& Francis Group, 2008. 314p.

PROGRAMA DE LAS NACIONES UNIDAS PARA EL DESARROLLO - PNUD. (2014). Informe sobre Desarrollo Humano 2014. Sostener el Progreso Humano: reducir vulnerabilidades y construir resiliência. Lançado em 24 de julho de 2014, em Tóquio, no Japão. Disponível em: $<$ http://www.pnud.org.br/arquivos/RDH2014es.pdf $>$. Acesso em: 03 set. 2014.

REIS, J. T.; SILVA, J. S.; MICHEL, G.P.; KOBIYAMA, M. Mapeamento da vulnerabilidade a desastres hidrológicos nos municípios de Alto Feliz e São Vendelino/RS como forma de contribuição à Engenharia de Sedimentos. In. XI Encontro Nacional de Engenharia de Sedimentos, João Pessoa: ABRH, 2014. 17p.

SCHENKEL, J.C.; MICHEL, G.P.; KOBIYAMA, M. Mapeamento de área de risco de escorregamentos translacionais na bacia do arroio Forromeco ๑ RS. In: Congresso Internacional de Hidrossedimentologia, Porto Alegre, Anais, 2015. (no prelo)

SOUZA, V. R.; GAVENAGHI, S.; ALVES, J.E.D. (2006). Mapeamento dos óbitos por local de ocorrência dos acidentes de trânsito na cidade do rio de Janeiro. In: Anais do XV Encontro Nacional de Estudos Populacionais. Caxambu: ABEP, 2006. 22 p. Disponível em: <http:// www.abep.nepo.unicamp.br/encontro2006/docspdf/ abep2006_358.pdf >. Acesso em: 13 out. 2014. 